\title{
3D Elemental Mapping in Nanomaterials by Core-Loss EFTEM Tomography
}

\author{
N.Y. Jin-Phillipp, ${ }^{*}$ C.T. Koch, ${ }^{*}$ and P. A. van Aken* \\ * Stuttgart Center for Electron Microscopy, Max Planck Institute for Metal Research, Heisenbergstr. 3, \\ 70569 Stuttgart, Germany
}

Having a complete 3-dimensional picture of a nanomaterial is often essential for understanding its properties. In contrast to biological specimen, bright-field image contrast from crystalline samples relevant to materials science depends strongly on the diffraction condition of the crystal, and can therefore generally not be used for tomography. Energy-filtered TEM (EFTEM) is an alternative imaging mode which overcomes this limitation [1,2], provided that multiple inelastic scattering can be neglected and that the dynamical (multiple elastic scattering) contrast is not too high. Using coreloss electrons to form an image, EFTEM tomography is element specific, and therefore may provide 3D compositional information, in addition to the shape information obtained by conventional tomography. In this paper, we will report 3D EFTEM tomography of iron-filled multi-walled carbon nanotubes (CNTs) and copper whiskers.

Tilt-series of EFTEM images were acquired on a Zeiss EM912 Omega microscope operated at $120 \mathrm{kV}$ using the Gatan EFTEM tomography plug-in for Digital Micrograph. The core-loss elemental maps were obtained by the three-window method (acquisition of 2 pre-edge images for extrapolating the background which was then subtracted from the post-edge image). The alignment of the elemental map tilt series by cross-correlation and the reconstructions using the simultaneous iterative reconstruction technique (SIRT) were performed with the FEI software package Inspect3D.

A 2D image combining $\mathrm{C}-\mathrm{K}$ and Fe- $\mathrm{L}_{23}$ elemental maps of a CNT filled with Fe is shown in Fig.1 (a). Tilt-series of both $\mathrm{C}-\mathrm{K}$ and $\mathrm{Fe}-\mathrm{L}_{23}$ EFTEM images were acquired at angles ranging from $+60^{\circ}$ to $-60^{\circ}$ in $2^{\circ}$ steps. The volume marked by the white rectangle in Fig. 1(a) was reconstructed by the SIRT algorithm with 10 iterations. Fig. 1(b), (c), and (d) show three orthogonal views of the 3D reconstruction, where the $x-y$ plane is the image plane at $0^{\circ}$ tilt. Interestingly, the CNT is revealed to be incomplete at its top, the Fe catalyst particle seems not to be enclosed. An extended EELS study shows that the crown of the catalyst particle contains oxygen and the iron there is oxidized. These results may be useful for understanding the growth of the CNTs, as well as their applications.

For the copper whisker tilt-series of both $\mathrm{C}-\mathrm{K}$ and $\mathrm{Cu}-\mathrm{L}_{23}$ EFTEM images were acquired at angles ranging from $+60^{\circ}$ to $-50^{\circ}$ in $2^{\circ}$ steps. $2 \mathrm{D}$ elemental maps of the $\mathrm{C}-\mathrm{K}$ and $\mathrm{Cu}-\mathrm{L}_{23}$ edges at $0^{\circ}$ tilt are shown in Fig.2 (a). The top part of the whisker was reconstructed using SIRT. Fig. 2(b) and 2(c) show two orthogonal views of the 3D reconstruction. The carbon layer covering the $\mathrm{Cu}$ whisker forms presumably during whisker growth. Though being obscured by the elongation along $z$ and reconstruction artifacts, the 6-fold symmetry of the cross-section of the whisker may be seen clearly from the isosurface of $\mathrm{Cu}$ viewed along the whisker (Fig.2 (d)).

Increasing the accuracy of the 3D elemental mapping requires improved image acquisition, better alignment of the tilt series and the three EFTEM images used for obtaining the elemental maps at each tilt angle, as well as better reconstruction methods. In order to reduce the dynamical contrast in the EFTEM images, conical illumination has been applied. The diffraction contrast in the elemental 
maps of $\mathrm{Fe}$ (in the $\mathrm{CNTs}$ ) and $\mathrm{Cu}$ (in the $\mathrm{Cu}$ whiskers) is significantly reduced by this technique. In some cases more accurate elemental maps were obtained by aligning the three EFTEM images manually. In order to allow a more quantitative interpretation of the EFTEM tomography reconstructions we also perform an approximate multiple (inelastic) scattering correction using the zero-loss image [3].

\section{References}

[1] P.A. Midgley and M. Weyland, Ultramicroscopy 96 (2003) 413.

[2] C. Kübel, et al., Microsc. Microanal. 11 (2005) 378.

[3] The authors acknowledge financial support from the European Union under the Framework 6 program under the contract for an Integrated Infrastructure Initiative Reference 026019 ESTEEM. They thank Kersten Hahn for his assistance and Nicole Grobert (Oxford University) and Gunther Richter for providing the CNTs and copper whiskers respectively.
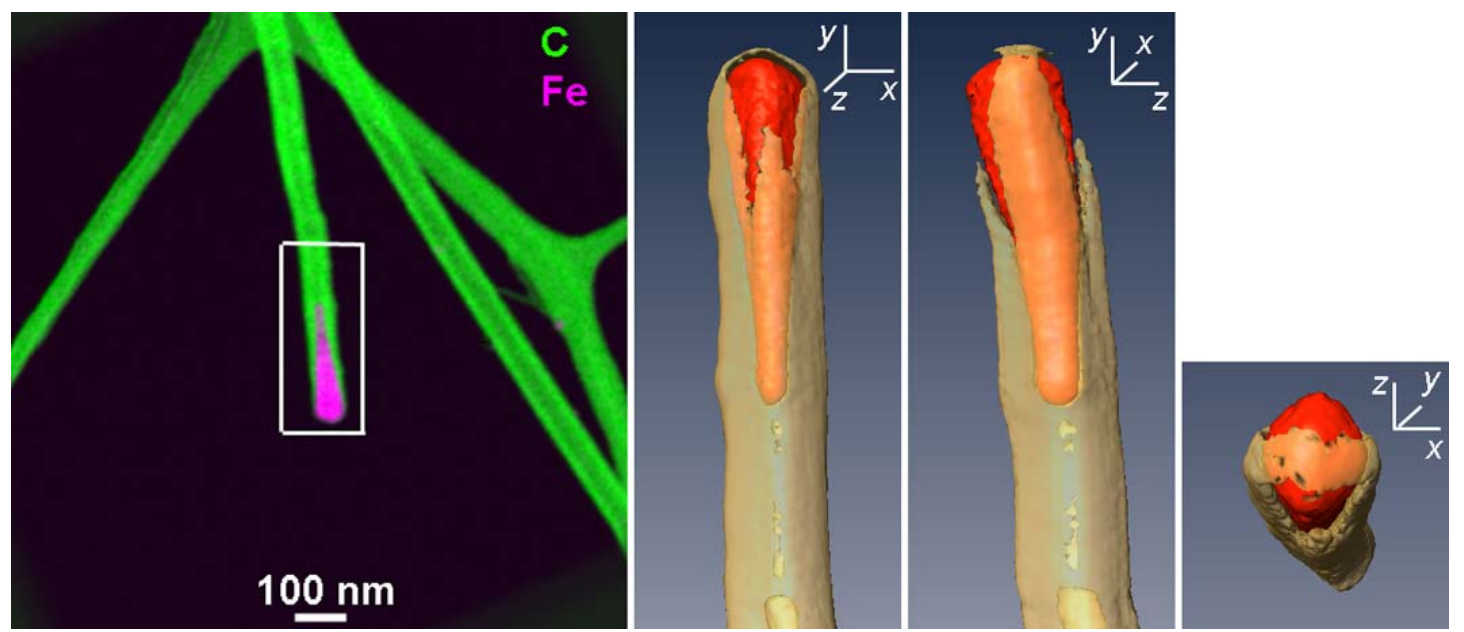

FIG. 1. (a) $\mathrm{C}-\mathrm{K}$ (green) and Fe- $\mathrm{L}_{23}$ (purple) elemental maps of an Fe-filled $\mathrm{CNT}$ at $0^{\circ}$ tilt. The reconstruction volume is marked by the white rectangle. (b)-(d) Three orthogonal views of the Fe filled nanotube reconstructed by SIRT, where $x-y$ is the image plane at $0^{\circ}$ tilt. Note the slight elongation along $z$, parallel to the direction of the incident electron beam due to the missing wedge.

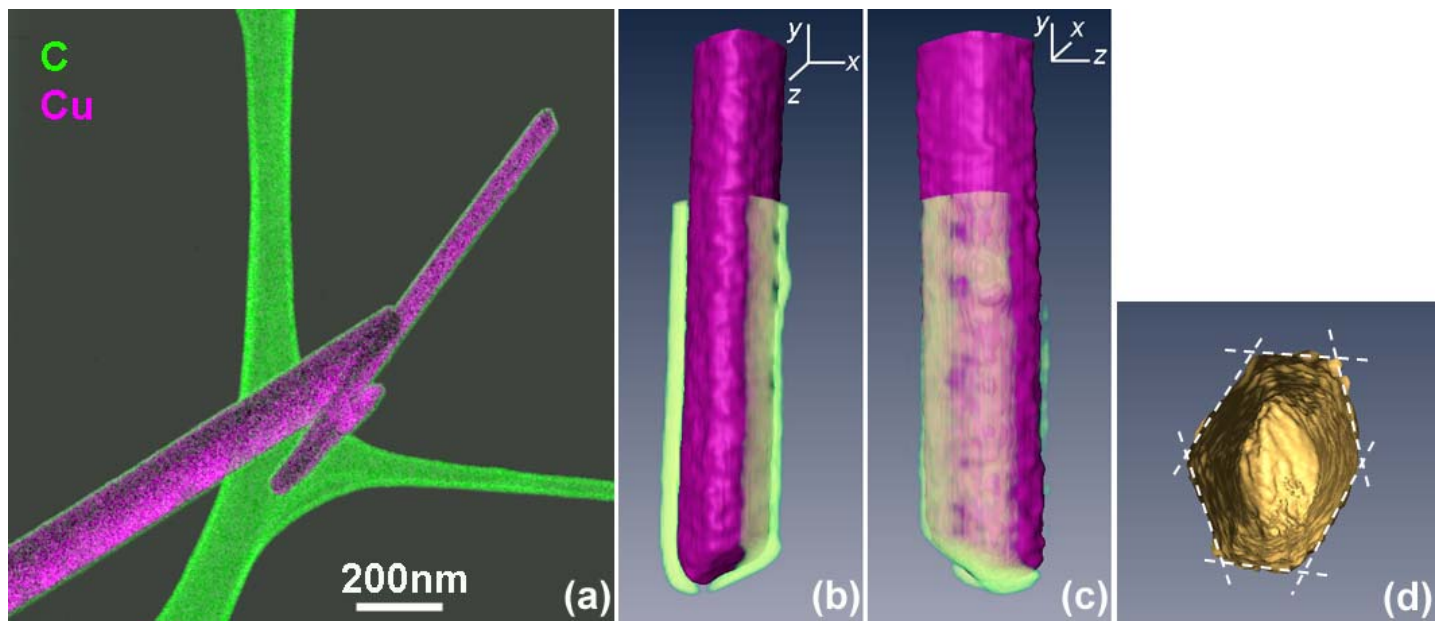

FIG. 2. (a) C-K (green) and $\mathrm{Cu}-\mathrm{L}_{23}$ (purple) elemental maps of a copper whisker at $0^{\circ}$ tilt. The top part of the whisker is reconstructed. (b) and (c) SIRT reconstruction viewed at two orthogonal directions, (d) The isosurface of $\mathrm{Cu}$ (enlarged) viewed along the whisker. 